\title{
Heterodyning Time Resolution Boosting for Velocimetry and Reflectivity Measurements
}

\author{
D.J. Erskine
}

This article was submitted to

SPIE Conference on Highspeed Photography and Photonics, Alexandria VA, September 19-21, 2004 SPIE Conference \#5580

\section{August 22, 2004}

U.S. Department of Energy

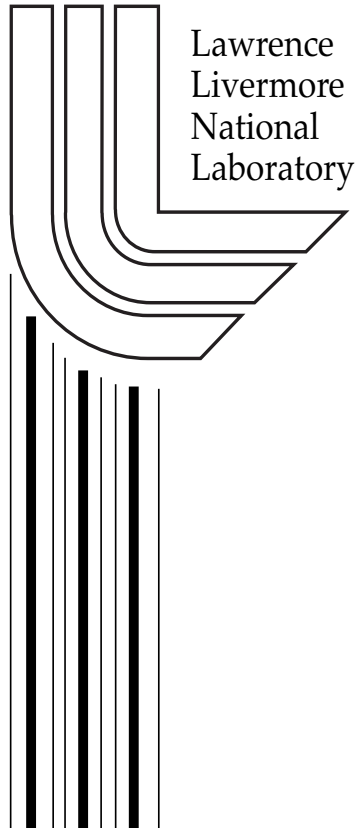


This document was prepared as an account of work sponsored by an agency of the United States Government. Neither the United States Government nor the University of California nor any of their employees, makes any warranty, express or implied, or assumes any legal liability or responsibility for the accuracy, completeness, or usefulness of any information, apparatus, product, or process disclosed, or represents that its use would not infringe privately owned rights. Reference herein to any specific commercial product, process, or service by trade name, trademark, manufacturer, or otherwise, does not necessarily constitute or imply its endorsement, recommendation, or favoring by the United States Government or the University of California. The views and opinions of authors expressed herein do not necessarily state or reflect those of the United States Government or the University of California, and shall not be used for advertising or product endorsement purposes.

This work was performed under the auspices of the U.S. Department of Energy by University of California, Lawrence Livermore National Laboratory under Contract W-7405-Eng-48.

Available from

Lawrence Livermore National Laboratory

Technical Information Department's Digital Library

http://www.llnl.gov/library/ 


\title{
Heterodyning Time Resolution Boosting for Velocimetry and Reflectivity Measurements
}

\author{
David J. Erskine \\ Lawrence Livermore Nat. Lab., 7000 East Ave, Livermore, CA 94550
}

\begin{abstract}
A theoretical technique is described for boosting the temporal resolving power by several times, of detectors such as streak cameras in experiments that measure light reflected from or transmitted through a target, including velocity interferometer (VISAR) measurements. This is a means of effectively increasing the number of resolvable time bins in a streak camera record past the limit imposed by input slit width and blur on the output phosphor screen. The illumination intensity is modulated sinusoidally at a frequency similar to the limiting time response of the detector. A heterodyning effect beats the high frequency science signal down a lower frequency beat signal, which is recorded together with the conventional science signal. Using 3 separate illuminating channels having different phases, the beat term is separated algebraically from the conventional signal. By numerically reversing the heterodyning, and combining with the ordinary signal, the science signal can be reconstructed to better effective time resolution than the detector used alone. The effective time resolution can be approximately halved for a single modulation frequency, and further decreased inversely proportional to the number of independent modulation frequencies employed.
\end{abstract}

Keywords: velocity interferometry, Doppler, VISAR, heterodyning, streak cameras

\section{INTRODUCTION}

An important kind of measurement performed at national laboratories and some industrial research laboratories is the transit time of a shockwave or high pressure pulse passing through a thickness of material. This determines the speed of the wave, which in turn determines the material equation of state. Accurate equation of state data is needed to resolve computational material models which often differ only by a slight degree.

The shock arrival time is typically measured by a sudden change in sample reflectivity when illuminated by an essentially constant intensity (long laser pulse) and recorded by a streak camera. Measurements of the material velocity through the Doppler shift of monochromatic illumination are similarly performed, often in the same experiment, using a velocity interferometer whose phased outputs are recorded by a streak camera.

The recording device of choice is often a streak camera since this provides many parallel input channels, usually assigned to spatial location across a target. A significant limitation to the common streak camera is the maximum number of resolvable elements in the phosphor screen which presents the streak, and which sets the maximum number of resolvable time elements in the record, no matter what the sweep speed or time duration of the record. Typically there are not more than 200 resolvable time bins, and will be less when used with increasing input slit width. Other streak camera defects include warping and nonlinear deviations of the electronic writing/streaking process which threaten accuracy.

The heterodyning technique described below effectively increases the number of resolvable time bins along the record, by decreasing the time resolution element while maintaining the same the record duration.

Further author information:

D.J.E.: E-mail: erskine1@1lnl.gov, Telephone: 19254229545 


\section{Heterodyning Reflectivity Measurement}

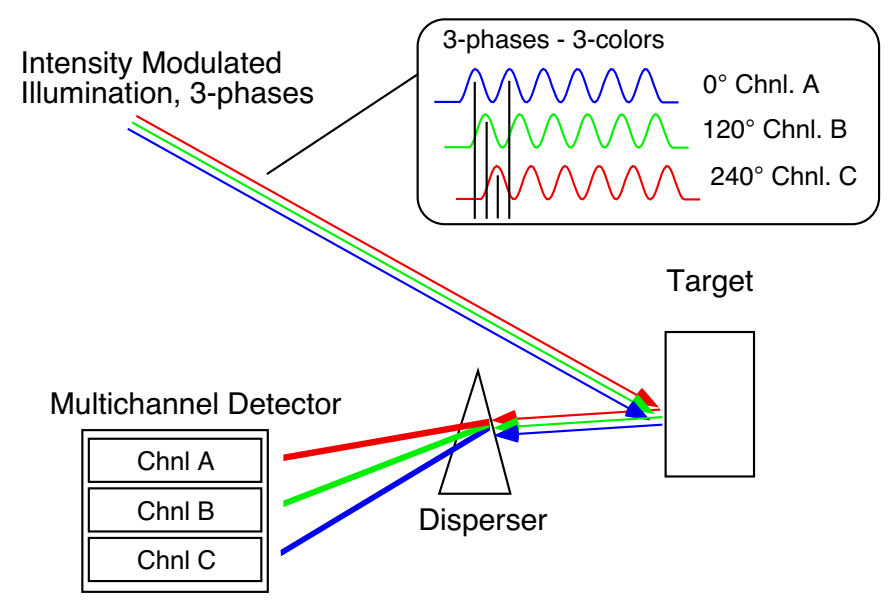

Figure 1. Heterodyning technique for measuring reflectivity at improved time resolution. This Figure shows only one point on the target, for simplicity. The illumination intensity is modulated sinusoidally at a frequency $f_{M}$. This causes reflectivity phenomena at frequency $f$ to be heterodyned to lower frequency $\left(f-f_{M}\right)$, forming a Moire signal recorded by the detector. The moiré signal is restored to the original frequencies during analysis. To permit algebraic separation between the ordinary and Moire components, which are embedded together in the recorded signal, the illumination is subdivided into 3 or 4 channels (a, b, c) have different phases separated by 120 or $90^{\circ}$. Here the phased channels are distinguished by wavelength. The channels can also be encoded by angle of incidence and/or polarization.

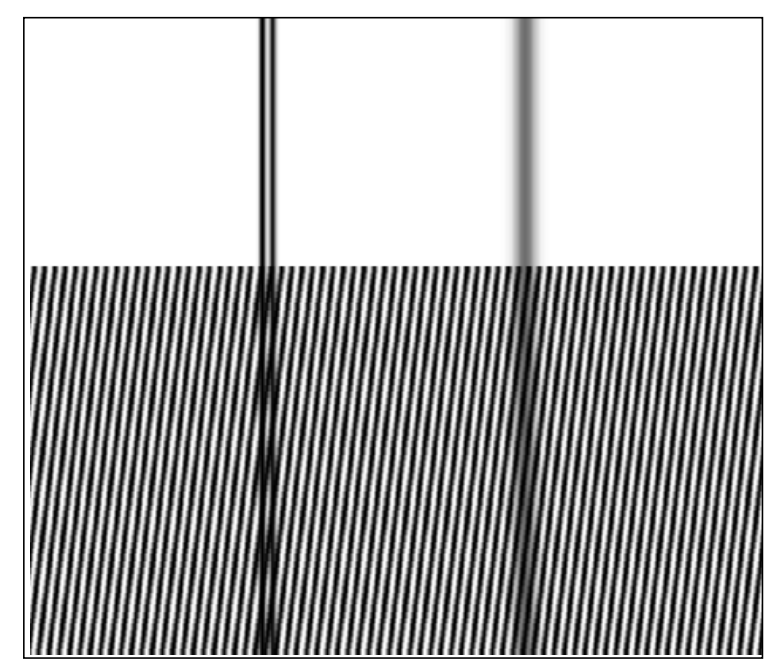

Figure 2. Graphical demonstration of the increased resolving power coming from a heterodyning effect. The classic definition of resolving power is ability to distinguish a doublet. If viewed from a distance, the doublet of lines on the left in the upper panel appears indistinguishable from the single line on the right. On the bottom panel a sinusoidal pattern is superimposed. The presence of the generated moiré pattern immediately distinguishes the doublet, hence the resolution has improved. 


\subsection{Heterodyning Measurement Technique}

A heterodyning technique (Fig. 1) is described here for improving the time resolution of such measurements, recorded by a multichannel detector (at least 3 channels). The technique can be employed for both reflectivity and VISAR velocity interferometer measurements. We will focus the discussion here to reflectivity measurements, for concreteness. Figure 1 depicts the technique applied to a reflectivity measurement. The apparatus differs from the ordinary reflectivity measurement by use of illumination $I(t)$ having periodic intensity modulation at a frequency $f_{m}$ or period $T_{m}=1 / f_{m}$.

$$
I(t) \propto\left[1+\cos \left(2 \pi t f_{m}\right)\right]
$$

A science signal having high frequency components in the neighborhood of $f_{p}$ is multiplied by the sinusoidal variation of $I(t)$ to form beats at a lower frequency $\left(f_{p}-f_{m}\right)$. These are more easily detected by the detector in spite of its limited frequency response $\left(f_{D} \approx 0.5 / T_{D}\right)$, where $T_{D}$ is its response time.

The beat generation effect, or "heterodyning" can be illustrated graphically in Figures 2 and 3 . Figure 2 demonstrates a temporal resolution boosting effect using the classical definition of resolving a pair of closely spaced lines. Note the appearance of a moiré pattern that distinguishes the doublet from the singlet. This shows that the technique can work for an arbitrary signal. Figure 3 shows that a similar moiré pattern can be obtained from the edge of a step function, which is a model for shock-like behavior. (The term "shock" is used loosely in this manuscript, not implying a perfectly discontinuous step.)

\subsubsection{Phased Illumination}

Let the illumination intensity for the $n^{\text {th }}$ channel be

$$
I_{n}(t) \propto\left[1+\cos \left(2 \pi t f_{m}+\phi_{n}\right)\right]
$$

where $\phi_{n}$ is the illumination channel phase. The $\phi$ is ideally evenly distributed around the phase circle. For 3 channels $\phi$ is 0,120 and $240^{\circ}$, and for 4 channels $0,90,180$ and $270^{\circ}$. The independent channels could be encoded by wavelength, polarization, angle of incidence, spatial location on target (provided target has spatially uniform behavior), or some combination thereof. Figure 7 shows a method of encoding the channels using wavelength.

By measuring data simultaneously in at least three separate channels where the illumination phase is different, it is possible to directly separate the ordinary and beat components arithmetically. (At least three channels are needed to unambiguously determined phase and magnitude of the beat component.) Removing the ordinary component is necessary prior to numerically reversing the heterodyning action, otherwise a portion of the ordinary signal will be inadvertently shifted to higher frequency where it will produce a false artifact. Hence multi-phased illumination is required.

Doppler velocimetry can also be performed simultaneously to measuring the target reflectivity. This is done by passing the reflected light from the target through an interferometer (often called a VISAR, for velocity interferometer system for any reflector $\left.{ }^{1}\right)$. The interferometer has multiple phased outputs, such as four in a push-pull system, ${ }^{2}$ or even more when the interferometer phase is made to vary spatially across a line across the target, which is then measured by a streak camera.

When making a VISAR measurement, the target reflectivity is measured "for free" along with the velocity. If the phased output of the VISAR is considered to be a complex signal, then the time varying magnitude is the sample reflectivity (times the illumination intensity) and the phase is proportional to the Doppler velocity. Hence, velocity interferometry measurement can be made with the same technique described here using multi-phased illumination, simply by treating the signal coming out of the interferometer, including after the heterodyning has been reversed, as complex.

However, the interesting point is that single-phased illumination, in addition to multi-phase illumination, can also be used to perform heterodyning velocity interferometry. Having only one illumination channel greatly simplifies the hardware. Having only a single channel of illumination is possible because the velocity interferometer provides the needed multiple phases as outputs (hence the multiphasing occurs after the target instead of prior to the target). However, the mathematics is not exactly analogous to the former case, and the separation of ordinary and beat components is not directbut can be solved with iteration. Details of this will be left to a future article.) 


\subsection{Benefits}

\subsubsection{Improved time resolution}

Figure 4 shows the 1-d results of a numerical simulation measuring a perfectly sharp step, with and without heterodyning, using a single modulation frequency. The risetime has approximately doubled. Figure 5 shows the boosted frequency response obtained using the heterodyning using a single frequency modulation, and Fig. 6 with $K$ multiple modulation frequencies. The temporal resolving power (proportional to the frequency response, and reciprocal of the time resolution element), is boosted by a factor $\sim(2 K+0.5)$.

\subsubsection{Robustness to streak sweep speed variations}

The sinusoidal modulations are built-in time fiducial markers that allow the measurement to be independent from sweep speed variations and other display distortions of the detector, that often occur with streak cameras. The responsibility for linearity now is removed from the detector and transferred to the illumination side of the problem.

\section{THEORY}

\subsection{Conventional detection}

If $S(t)$ is the target reflectivity and $I(t)$ the illumination history, then the signal reaching the detector is $R(t)=S(t) I(t)$. For the conventional method $I(t)=1$. The detected ordinary signal $B_{\text {ord }}(t)$, which is the blurred version of $R(t)$ and hence $S(t)$, is computed by a convolution with the detector impulse response $D(t)$,

$$
B_{\text {ord }}(t)=S(t) \otimes D(t)
$$

The convolution is more conveniently expressed as a multiplication in Fourier-space,

$$
\mathbf{b}_{\text {ord }}(f)=\mathbf{s}(f) \mathbf{d}(f)
$$

where lower case symbols are the Fourier transformed (fft) versions of the respective functions. The $\mathbf{d}(f)$ is the detector frequency response $\mathbf{d}(f) \equiv \mathrm{fft} D(t)$. For simplicity we model $d(f)$ and $D(t)$ as Gaussians having full widths at half max (FWHM) of $f_{D}$ and $T_{D}$. We desire narrow $D(t)$ and broad $d(f)$.

Figure 5a show that the instrument response for the conventional technique is a peak centered at zero frequency, having limited ability to detect higher frequency components. High frequency is often the region of most interest in a typical science signal. (The narrower the rise of a step function the higher the frequency of its components.)

\subsection{Heterodyning detection}

In contrast to the constant intensity of the conventional method, the heterodyning technique has each of several illumination channel intensities modulated sinusoidally

$$
I_{n}(t)=1+\gamma \cos \left(2 \pi t f_{M}+\phi_{n}\right),
$$

where $\gamma$ is the modulation degree and $\phi_{n}$ is the phase of each channel. Three or four blurred signals $B_{n}(t)$ are recorded on the streak camera or detector having multiple channels at phases $\phi_{n}$ differing by $120^{\circ}$ or $90^{\circ}$. It is useful to employ an alternative designation for the detected channels using the phase in degrees as the subscript, $B_{0}, B_{90}, B_{180}$ etc. Generically, any number of phase channels greater than two can be used, ideally evenly spaced around the phase circle so that the vector sum is zero. Three is the minimum needed to unambiguously separate the beat from ordinary signals.

The detected signal $B_{n}(t)$ for the heterodyning technique is Equation 3 modified to include the time dependence of $I(t)$, which is no longer constant.

$$
B_{n}(t)=\left[S(t) I_{n}(t)\right] \otimes D(t) .
$$

Substitution of Eq. 5 for $I(t)$ forms a sum of the ordinary measured signal plus two beat or moiré terms.

$$
\begin{aligned}
B_{n}(t)= & B_{\text {ord }}(t)+\frac{1}{2}\left[S(t) e^{i \phi_{n}} e^{i 2 \pi t f_{M}}+\right. \\
& \left.S(t) e^{-i \phi_{n}} e^{-i 2 \pi t f_{M}}\right] \otimes D(t) .
\end{aligned}
$$



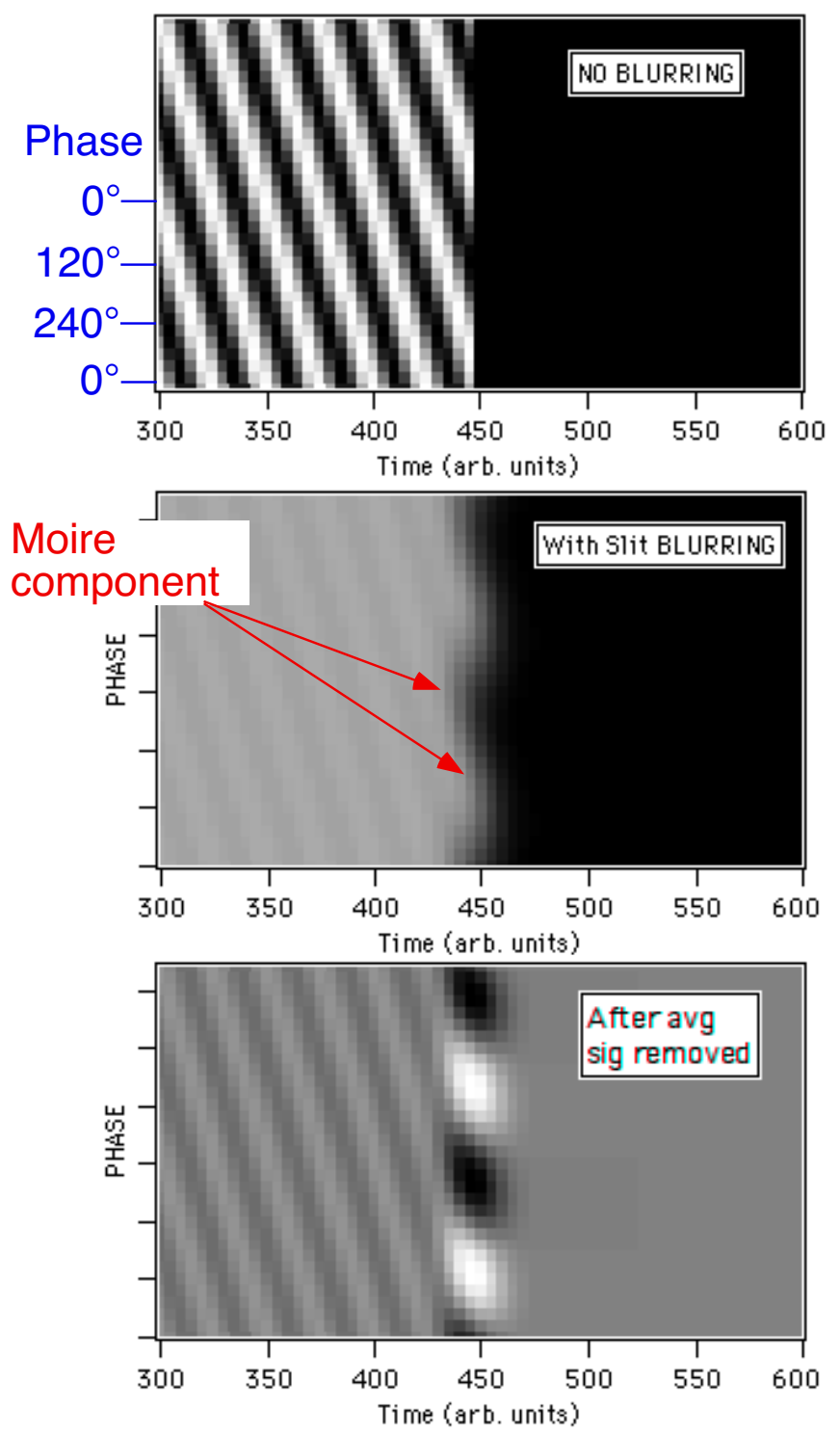

Figure 3. Graphical demonstration that a moiré signal can be generated from a sharp edge. Simulated streak camera recording of a sudden change in reflectivity of a target illuminated by a periodically modulated illumination intensity. (a) Blurring is ignored. The modulation phase is plotted vertically so that all phases can be seen at once. (b) After detector blurring is imposed; note the wavy edge of the step, which is the moiré or beat component. (c) Removing the average signal highlights the moiré component. Its phase accurately measures the reflectivity edge location in spite of detector distortions, since the illumination and science signal distort together. 


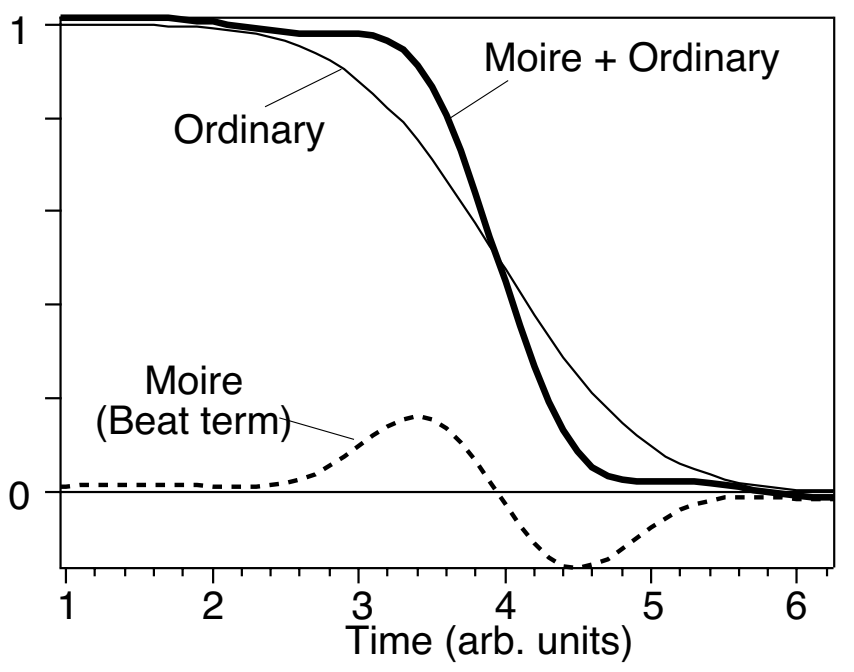

Figure 4. Numerically simulated result of measuring a perfectly sharp step function with a detector having a finite response time, without (thin curve, "Ordinary") and with (bold, "Moire+Ordinary") sinusoidal modulation. By adding the moiré (beat) component to the ordinary the measured risetime is approximately twice as fast.
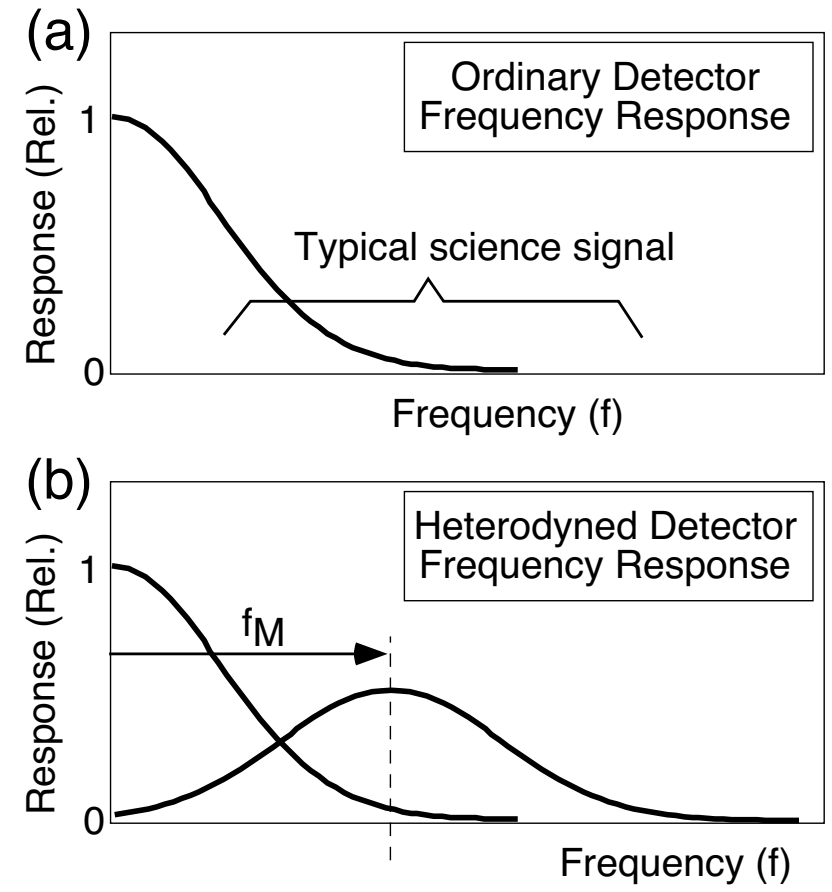

Figure 5. Idealized frequency response of the (a) conventional measurement and (b) heterodyning measurement. The most interesting portion of a typical science signal is often in the highest frequencies, which is where a detector is pushed to its performance limit. In the heterodyning technique (b) the illumination modulation creates a sideband in the response, which has the same shape as the ordinary response but shifted to higher frequency by $f_{M}$, (and one half the height). By choosing $f_{M}$ to be at the shoulder of the ordinary response, the net frequency response is effectively extended. 


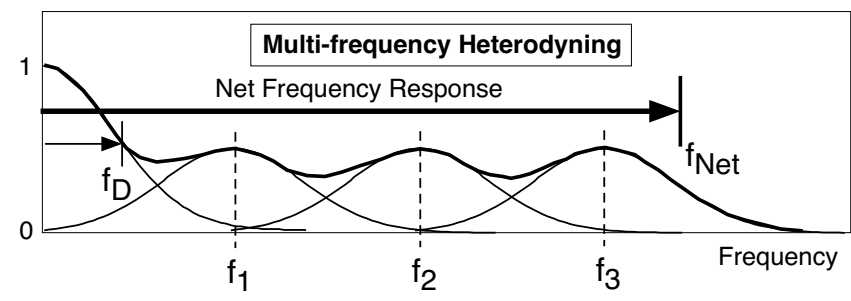

Figure 6. Frequency response when $K$ multiple modulating frequencies $\left(f_{1}, f_{2}, f_{3}\right)$ are employed in parallel. The net frequency response $f_{\text {net }}$ is boosted over the conventional frequency response $\left(f_{D}\right)$ by a factor $\Psi=f_{N e t} / f_{D} \sim(2 K+0.5)$.

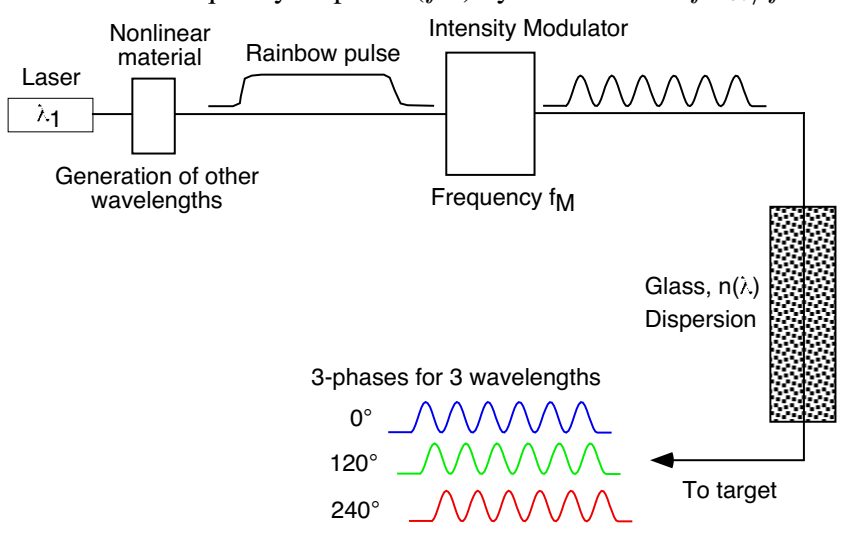

Figure 7. A method for making multi-phased illumination encoded by wavelength. An intense laser pulse passing through a nonlinear material generates a variety of wavelengths, all having similar duration. This broadband pulse passes through an intensity modulator where the same sinusoidal modulation at frequency $f_{M}$ is imprinted on each. Passage through a dispersive medium such as glass delays shorter wavelengths relative to longer due to $\lambda$ dependence of refractive index $n$. Glass length is chosen so that the delay (relative to $1 / f_{M}$ ) between 3 distinguishable wavelengths is $120^{\circ}$ of phase shift.

We obtain one stationary, one clockwise, and one counter-clockwise rotating term, versus $\phi$.

The beat component is isolated from the ordinary component $B_{\text {ord }}$ by taking a set of $N$ phase shifted data and forming a complex linear combination called a "whirl", $\mathbf{W}(t)$, where $B_{n}$ are numerically anti-rotated to undo each channel's optically imposed phase shift. The whirl in general for $N$ phase channels is

$$
\mathbf{W}(t)=\frac{1}{N} \sum B_{n} e^{i \phi_{n}}
$$

For four phase recordings every $90^{\circ}$ the whirl is particularly simple:

$$
\begin{aligned}
\mathbf{W}(t) & =\frac{1}{4}\left(B_{0} e^{i 0^{\circ}}+B_{90} e^{i 90^{\circ}}+B_{180} e^{i 180^{\circ}}+B_{270} e^{i 270^{\circ}}\right) \\
& =\frac{1}{4}\left[\left(B_{0}-B_{180}\right)+i\left(B_{90}-B_{270}\right)\right] .
\end{aligned}
$$

The whirl for 3 phase channels is

$$
\begin{aligned}
\mathbf{W}(t) & =\frac{1}{3}\left(B_{0} e^{i 0^{\circ}}+B_{120} e^{i 120^{\circ}}+B_{240} e^{i 240^{\circ}}\right) \\
& =\frac{1}{6}\left[\left(2 B_{0}-B_{120}-B_{240}\right)+i \sqrt{3}\left(B_{120}-B_{240}\right)\right] .
\end{aligned}
$$

Applying Eq. 8, 9, or 10 to Eq. 7 we get

$$
\mathbf{W}(t)=\frac{1}{2}\left[e^{i 2 \pi t f_{M}} S(t)\right] \otimes D(t) .
$$

This expression in the frequency domain is

$$
\mathbf{w}(f)=\frac{1}{2} \gamma \mathbf{s}\left(f+f_{M}\right) \mathbf{d}(f)
$$


where we include the modulation degree $(\gamma)$ previously taken as unity.

This important equation describes the heterodyning formation of the moire or beat signal. The heterodyning is the shifting expressed in the $\mathbf{s}\left(f+f_{M}\right)$ argument. Fine temporal details in the science signal $s(f)$ having high frequency $f$ are shifted by $f_{M}$ to low frequency prior to blurring by $\mathbf{d}(f)$. This effectively shifts the sensitivity of the detector to higher frequencies by $f_{m}$, as depicted by Fig. 5 b.

\subsubsection{Extracting the ordinary spectrum}

Note that even though the illumination is modulated, the ordinary unmodulated signal is easily obtained from the data by a straight sum of the phase-stepped data (so that oscillatory terms cancel),

$$
B_{\text {ord }}(t)=\frac{1}{N} \sum B_{n}
$$

or specifically for 3 or 4 phase channels:

$$
\begin{array}{r}
B_{\text {ord }}(t)=\frac{1}{3}\left(B_{0}+B_{120}+B_{240}\right) \text { or } \\
B_{\text {ord }}(t)=\frac{1}{4}\left(B_{0}+B_{90}+B_{180}+B_{270}\right) .
\end{array}
$$

\subsection{Signal Recovery and Response}

To recover $S(t)$ at a improved time resolution, the measured beat signal $\mathbf{w}(f)$ is processed to reverse the heterodyning expressed in Eq. 12. This is then combined with the ordinary signal to form a composite output. This is analogous to combining the "bass" and "treble" channels of an audio system to obtain a more full-bodied sound.

The data processing steps include (1) resampling $\mathbf{W}(t)$ to linearize vs $t$, so that in the next step the modulation comb component forms a very narrow spike in $\mathbf{w}(f)$ at $f_{M}$. (2) Fourier transform $\mathbf{W}(t)$ to form $\mathbf{w}(f)$ and translate by $f_{M}$ toward higher $f$. Hence low frequency detected moiré components become high frequency signals. (3) Delete the negative branch, which does not hold as significant of signal and is noisier than the positive branch. (4) Inverse Fourier transform and take the real part to form the "treble" signal $B_{h e t}(t)$. (5) Sum the treble and ordinary signals to form a composite signal, after first weighting the components by an amount proportional to their expected strength vs $f$. This discriminates against noise for frequencies where the signal is known to be noisy. (6) Equalize the frequency distribution of the composite signal to force the instrument lineshape into a user-desired response such as Gaussian, which minimizes ringing. The weighting function needed for equalization is obtained from a calibration recording on a known signal, which calibrates the detector blurring $d(f)$. Further discussion of this data analysis procedure is in Ref. 3.

\section{METHODS OF ENCODING PHASED ILLUMINATION}

Figure 7 shows a method of encoding the channels using wavelength. A laser generates a long pulse (sufficiently long to cover the duration of the measurement). This is passed through some nonlinear optical material to generate other wavelengths besides the original laser wavelength. Depending on details of the time scale of the pulse and its intensity, it might be possible to generate a white light pulse, or use 2nd and 3rd harmonic generation. For longer time scales (where the intensity may not be sufficient for nonlinear optics), the single laser could be replaces by 3 lasers operating at different wavelengths but synchronized to produce a pulse simultaneously and having the same duration. In any case, the multiwavelength pulse then passes through a device for modulating its intensity (discussed below). Finally, the phases of the multiple wavelengths are shifted by delaying one wavelength relative to the other. For high $f_{M}$, only small delays are needed, and this could be done by the dispersion in ordinary transparent materials such as glass. For lower $f_{M}$ and thus longer delays, this could be done by separating the wavelengths with dichroic filters or a prism or grating and having the optical path length differ as a function of the wavelength, before it is ultimately recombined on the target.

\section{RELATED DEMONSTRATIONS}

Experimental demonstrations of this theoretical technique have not yet been performed in the time domain. However, demonstrations ${ }^{3-5}$ have been performed in the spectral domain, where the mathematics is analogous but the experimental hardware is of course different. In fact, the software developed for the spectral application was used in the numerical simulation of time resolution boosting shown in Figure 4, by substituting the time variable for the optical frequency of 
light (wavenumber, $1 / \lambda$ ) variable in the spectral demonstration. In both cases a moire process is occurring in the apparatus, which is reversed numerically during data analysis, that shifts the science signal to lower frequencies in the relevant variable so that it is easier to detect.

The spectral resolution of the Lick Observatory spectrograph was approximately doubled ${ }^{3}$ by imprinting a sinusoidal comb on starlight with an interferometer of fixed delay inserted into the beam, and postprocessing the data as described above. In recent experiments ${ }^{5}$ the spectral resolution of a benchtop grating spectrograph measuring the iodine spectrum was boosted approximately 6 times, from its native 25,000 resolution to an effective 140,000, over the full bandwidth of the spectrograph by using multiple heterodyning exposures and combining the individual results. These demonstrations show that reconstruction of a full-bodied data set from individual down-heterodyned channels is possible. The spectral heterodyning technique has been described by the author in a US patent. ${ }^{6}$

\section{ACKNOWLEDGMENTS}

This work was performed under the auspices of the U.S. Department of Energy by the University of California, Lawrence Livermore National Laboratory under contract No. W-7405-Eng-48.

\section{REFERENCES}

1. L. Barker and R. Hollenbach, "Laser Interferometer for Measuring High Velocities of any Reflecting Surface," J. Appl. Phys. 43, pp. 4669-4675, 1972.

2. W. Hemsing, "Velocity Sensing Interferometer (VISAR) Modification," Rev. Sci. Instr. 50, pp. 73-78, 1979.

3. D. Erskine, J. Edelstein, M. Feuerstein, and B. Welsh, "High Resolution Broadband Spectroscopy using an Externally Dispersed Interferometer," ApJ 592, pp. L103-L106, 2003.

4. D. Erskine and J. Edelstein, "High-resolution Broadband Spectral Interferometry," in Future EUV/UV and Visible Space Astrophysics Missions and Instrumentation, ed. J. C. Blades, O. H. Siegmund, pp. 158-169, SPIE Proc. 4854, Feb. 2003.

5. D. Erskine, "Interferometric Resolution Boosting for Spectrographs," in Ground-based Instrumentation for Astronomy, ed. A. Moorwood, SPIE Proc. 5492, June 2004.

6. D. Erskine, "Combined Dispersive/Interference Spectroscopy for Producing a Vector Spectrum," US Patent 6,351,307, Feb. 26, 2002. 